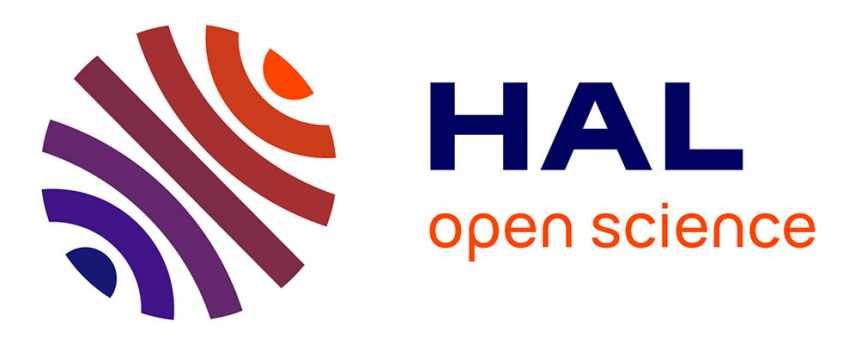

\title{
Liaisons unilatérales et chocs élastiques quelconques: un résultat d'existence
}

Mongi Mabrouk

\section{To cite this version:}

Mongi Mabrouk. Liaisons unilatérales et chocs élastiques quelconques: un résultat d'existence. Comptes Rendus de l'Académie des Sciences - Series I - Mathematics, 1998, 326 (11), 10.1016/S07644442(98)80193-6 . hal-01364846

\section{HAL Id: hal-01364846 \\ https://hal.science/hal-01364846}

Submitted on 13 Sep 2016

HAL is a multi-disciplinary open access archive for the deposit and dissemination of scientific research documents, whether they are published or not. The documents may come from teaching and research institutions in France or abroad, or from public or private research centers.
L'archive ouverte pluridisciplinaire $\mathbf{H A L}$, est destinée au dépôt et à la diffusion de documents scientifiques de niveau recherche, publiés ou non, émanant des établissements d'enseignement et de recherche français ou étrangers, des laboratoires publics ou privés. 


\title{
Liaisons unilatérales et chocs élastiques quelconques : un résultat d'existence
}

\section{Mongi MABROUK}

LMA, 24, rue de l'épitaphe, 25000 Besançon cedex, Francé

Courriel : mongi.mabrouk@univ-fcomte.fr

Résumé. Suivant J.J. Moreau [5], nous présentons un modèle variationnel unifié qui décrit l'évolution d'un système mécanique ayant un nombre fini de degrés de liberté, soumis à des liaisons unilatérales parfaites et subissant des chocs de coefficient de restitution quelconque. Dans le cas d'une seule contrainte $f(q) \leq 0$, nous montrons un résultat d'existence sous la condition que $f$ est de classe $\mathrm{C}^{1 \cdot \sqrt{3}}$, avec $\beta>1 / 2$.

\section{Unilateral constraints and arbitrary elastic shocks: an existence result}

\begin{abstract}
Following J.J. Moreau [5], we present a unified variational model, which governs the evolution of a mechanical system having a finite number of degrees of freedom, submitted to some perfect unilateral constraints, and experiencing shocks with arbitrary restitution coefficient. In the case of one single constraint $f(q) \leq 0$, we prove an existence result, under the assumption that $f$ is of class $\mathrm{C}^{1.3}$, with $\beta>1 / 2$.
\end{abstract}

\section{Abridged English Version}

We consider a mechanical system with a finite number of degrees of freedom, having a configurations manifold $Q$ and a local coordinates system $q=\left(q_{1}, q_{2}, \ldots, q_{N}\right)$. Besides the bilateral constraints which have been used for the parametrization of $Q$, we consider a single perfect unilateral constraint $f(q) \leq 0$, where $f$ is at least $\mathrm{C}^{1}$, with $\nabla f \neq 0$ in a neighborhood of the hypersurface $f(q)=0$ delimiting the closed domain $L \subset Q$. Let $E(q)$ (resp. $E^{\prime}(q)$ ) be the tangent (resp. cotangent) space at $q$. To avoid unnecessary complications, we suppose that the kinetic energy reduces to $T(q, \dot{q})=\frac{1}{2}\|\dot{q}\|^{2}$ which makes $Q$, at least locally, an open subset of $\mathbb{R}^{N}$ and $E(q)=E^{\prime}(q)=\mathbb{R}^{N}$. For every $q$, let $V(q)=\{w \in E: w \cdot \nabla f(q) \leq 0\}$ if $f(q) \geq 0$, and $V(q)=E$ if $f(q)<0$, which is the tangent cone to $L$ at $q$ if $q \in L$. Here $V(q)$ is either $\mathbb{R}^{N}$ or a half-space. Let $N(q)$ be the polar cone

Note présentée par Philippe G. Ciarlet. 
of $V(q)$. The system is submitted to some continuous given forces $p(t, q(t)) \in E^{\prime}(q)$ and to an unknown constraint force $R=-\lambda \nabla f(q), \lambda \geq 0$ constant. Let $I=[0, T] \subset \mathbf{R}$. Then a generalized motion is a locally absolutly continuous map $q: I \longrightarrow Q$ such that: i) the right and left velocities $\dot{q}^{ \pm}$exist everywhere, ii) on every subinterval of smooth motion we have: $p-\ddot{q} \in \partial I_{V(q)}(\dot{q})$ a.e., iii) if at an instant $t_{s}$, we have $\dot{q}^{-}\left(t_{s}\right) \neq \dot{q}^{+}\left(t_{s}\right)$, then a shock occurs and we have the jump relation: $\frac{1}{1+e}\left(\dot{q}^{+}+e \dot{q}^{-}\right)=\operatorname{prox}\left(V(q), \dot{q}^{-}\right), \operatorname{prox}(C, x)$ being the proximal point to $x$ in the convex set $C$.

DEFINITION. - A function $u$, locally of bounded variation on $I$ is said to be e-averaged, and denoted lbve-m, if and only $u=\frac{u^{+}+e u^{-}}{1+e}$ everywhere.

We can then state our problem:

Problem $\mathcal{P}$. - Let $q_{0} \in L, v_{0} \in E$. To find $u$ in $\operatorname{lbve-m}(I, Q)$ with $u^{-}(0)=v_{0}$ such that with $q(t)=q_{0}+\int_{0}^{t} u(\tau) \mathrm{d} \tau$ we have the following differential equation for real measures: $p(t, q(t)) \mathrm{d} t-\mathrm{d} u \in \partial I_{V(q(t))}(u(t))$.

From [1], we know that every solution $u$ of Problem $\mathcal{P}$ is a generalized motion. In the present work, we prove the following existence result by using the numerical scheme (6)-(9).

THEOREM. - Assume $f \in \mathrm{C}^{1, \beta}(Q, \mathbb{R}), \beta>1 / 2$. Then:

(i) from the sequence $\left(v_{n}, q_{n}\right)$ of the numerical sheme, we can extract a subsequence which converges to a limit $(v, q)$ where $v$ is of bounded variation and $q(t)=q_{0}+\int_{0}^{t} v(\tau) \mathrm{d} \tau$;

(ii) the function $u=\frac{v^{+}+e v^{-}}{1+e}$ is a solution of Problem $\mathcal{P}$.

The above result remains true if we assume that $p$ depends also on $\dot{q}$ (viscous damping for example) and is uniformly Lipshitzian in $q$ and $\dot{q}$.

\section{Présentation}

Dans ce travail, nous exposons et étudions un modèle variationnel unifié qui gouverne la dynamique des systèmes mécaniques à un nombre fini de degrés de liberté, soumis à des liaisons unilatérales parfaites. Ce modèle déjà mentionné par J.J. Moreau dans [5], s'applique en présence de chocs de nature quelconque, généralisant ainsi le cas du choc inélastique standard étudié initialement par J.J. Moreau [4]. Plus précisément, considérons un système mécanique à $N$ degrés de liberté. Soit $Q$ la variété des configurations et $q=\left(q_{1}, q_{2}, \ldots, q_{N}\right)$ un système de coordonnées locales. En plus des liaisons bilatérales qui ont servi à choisir la paramétrisation, on suppose le système soumis à une liaison unilatérale parfaite, d'équation :

$$
f(q) \leq 0,
$$

où $f$ est supposée de classe $C^{1}$ au moins, de gradient non nul au voisinage de l'hypersurface $f(q)=0$ délimitant la région fermée régulière $L \subset Q$. Soit $E(q)$ (resp. $E^{\prime}(q)$ ) l'espace tangent (resp. cotangent) en $q$ à $Q$. Afin d'éviter les complications purement techniques, nous supposons que l'énergie cinétique du système se réduit à $T(q, \dot{q})=\frac{1}{2}\|\dot{q}\|_{E}^{2}$, i.e. $Q$ est un ouvert de $\mathbb{R}^{N}$, et $E(q)=E^{\prime}(q)=\mathbb{R}^{N}$.

Soit $V(q)=\{w \in E: w \cdot \nabla f(q) \leq 0\}$ si $f(q) \geq 0$, et $V(q)=E$ si $f(q)<0$, appelé cône tangent à $L$ au point $q$ si $q \in L$. Ici $V(q)$ est soit l'espace entier, soit un demi-espace, et la condition de qualification $\operatorname{Int} V(q) \neq \emptyset$ est satisfaite pour tout $q$. Soit $N(q):=\{r \in E: r \cdot w \leq 0, \forall w \in V(q)\}$ le cône polaire à $V(q)$. Dans notre cas, $N(q)$ est la normale sortante à $L$ si $f(q)=0$, et $\{0\}$ si 
$f(q)<0$. Si le mobile $t \longmapsto q(t)$, décrivant l'évolution du système, reste dans la région $L$, on a : $v^{+} \cdot \nabla f(q) \leq 0$, où $v^{+}=\dot{q}^{+}$est la vitesse à droite de $q$ supposée exister. Autrement dit, $v^{+} \in V(q)$. On montre de même que si la vitesse à gauche $v^{-}$existe, on a $v^{-} \in-V(q)$. Du point de vue de la dynamique, le système est soumis à des efforts donnés $p(t, q(t)) \in E^{\prime}(q)$ continus et à des efforts de liaison représentés par le covecteur $R=-\lambda \nabla f(q), \lambda$ constante positive. Le mouvement $q: I \subset \mathbf{R} \longrightarrow Q$, où $I$ est un intervalle ouvert, est dit régulier si et seulement si $q$ est différentiable, et $\dot{q}$ est localement absolument continue. Si $I$ est un intervalle de mouvement régulier, les équations de Lagrange s'écrivent :

$$
p-\ddot{q} \in \partial I_{V(q)}(\dot{q})=N_{V(q)}(\dot{q}) \quad \text { p.p. }
$$

où $I_{C}$ est ici la fonction indicatrice du convexe $C$, égale à 0 sur $C$ et $+\infty$ sinon, et $\partial I_{C}(u)$ est son sous-différentiel au point $u$.

Si un intervalle de mouvement régulier se termine à $t_{s}$ avec $\dot{q}^{-}\left(t_{s}\right) \notin V\left(q\left(t_{s}\right)\right)$, on a nécessairement un choc. Notons $\pi=\dot{q}^{+}\left(t_{s}\right)-\dot{q}^{-}\left(t_{s}\right)$ la percussion de liaison.

DÉFINITION 1. - Soit $0 \leq e \leq 1$. Un choc à l'instant $t_{s}$ est dit élastique de coefficient de restitution $e$ (inélastique standard si $e=0$ ) si et seulement si l'une des quatre conditions équivalentes suivantes a lieu : (i) $\frac{1}{1+e}\left(\dot{q}^{+}+e \dot{q}^{-}\right)=\operatorname{prox}\left(V(q), \dot{q}^{-}\right)$, (ii) $-\frac{\pi}{1+e}=\operatorname{prox}\left(N(q), \dot{q}^{-}\right)$, (iii) $\frac{\pi}{1+e} \cdot\left(\dot{q}^{+}+e \dot{q}^{-}\right)=0$ ou (iv) $\frac{1}{2}\left\|\dot{q}^{+}\right\|_{E}^{2}-\frac{1}{2}\left\|\dot{q}^{-}\right\|_{E}^{2}=-\frac{1}{2} \cdot \frac{1-e}{1+e}\left\|\dot{q}^{+}-\dot{q}^{-}\right\|_{E}^{2}$, avec $\operatorname{prox}(C, x)$ désignant le point proximal de $x$ dans le convexe fermé $C$.

Pour $e=0$, on retrouve le choc inélastique (ou mou) étudié par J.J. Moreau [4] et P. MonteiroMarques [2]. Suivant J.J. Moreau, nous appellerons mouvement généralisé toute application $q: I \subset \mathbf{R} \longrightarrow Q$, localement absolument continue, ayant des dérivées à droite et à gauche $\dot{q}^{ \pm}$ en tout point, vérifiant les équations du mouvement régulier sur tout sous-intervalle $I^{\prime} \subset I$ où la dérivée $\dot{q}$ existe et est localement absolument continue, et vérifiant en tout point de discontinuité $t$, les conditions de choc.

DEFINITION 2. - Une fonction $u$, localement à variation bornée sur un intervalle $I \subset \mathbf{R}$, est dite e-moyennée, et sera notée lvbe-m, si et seulement si $u=\frac{u^{+}+e u^{-}}{1+e}$ en tout point de $I$.

On se pose alors le problème suivant :

Problème $\mathcal{P}$. - Soient $q_{0} \in L$ et $v_{0} \in E$. Trouver $u \in \operatorname{lvbe}-\mathrm{m}(I, E)$, avec $u^{-}(0)=v_{0}$, de telle sorte qu'en posant $q(t)=q_{0}+\int_{0}^{t} u(\tau) \mathrm{d} \tau$, on ait l'inclusion différentielle au sens des mesures réelles :

$$
p(t, q(t)) \mathrm{d} t-\mathrm{d} u \in \partial I_{V(q(t))}(u(t))
$$

On montre alors (voir [1]) que toute solution du problème $\mathcal{P}$ est un mouvement généralisé vérifiant une forme généralisée du théorème de l'énergie cinétique : $\forall t \in I: u(t) \cdot\left(p(t, q(t)) t_{\mu}^{\prime}(t)-u_{\mu}^{\prime}(t)\right)=0$, et est dissipative dans le sens : $\forall t \in I,\left\|u^{+}(t)\right\| \leq\left\|u^{-}(t)\right\|$. Dans ce travail, nous étudions la question de l'existence de solutions pour le problème $\mathcal{P}$. Pour cela, nous employons une technique de discrétisation analogue à celle utilisée par P. Monteiro-Marques dans [2]. Le résultat principal de cette Note est un théorème d'existence :

ThÉoRème. - Supposons $f \in \mathbb{C}^{1,3}(Q, \mathbb{R})$, avec $\beta>1 / 2$. Alors :

(i) de la suite $\left(v_{n}, q_{n}\right)$ du schéma numérique (6)-(9), on peut extraire une sous-suite convergeant vers une limite $(v, q)$ où v est une fonction à variation bornée sur $[0, T]$ et $q(t)=q_{0}+\int_{0}^{t} v(\tau) \mathrm{d} \tau$;

(ii) la fonction $u=\frac{u^{+}+e u^{-}}{1+e}$ est une solution du problème $\mathcal{P}$. 


\section{Démonstration du théorème}

Nous discrétisons la relation (3) sous la forme implicite :

$$
\frac{t_{i+1}-t_{i}}{1+e} p\left(t_{i+1}, q\left(t_{i+1}\right)\right)-\frac{v_{i+1}-v_{i}}{1+e} \in \partial I_{r^{r}\left(q\left(t_{i+1}\right)\right)}\left(\frac{v_{i+1}+e v_{i}}{1+e}\right)
$$

équivalente à :

$$
\frac{v_{i+1}+e v_{i}}{1+e}=\operatorname{prox}\left(V\left(q\left(t_{i+1}\right)\right), v_{i}+\frac{t_{i+1}-t_{i}}{1+e} p\left(t_{i+1} \cdot q\left(t_{i+1}\right)\right)\right)
$$

Nous allons esquisser la preuve de l'existence d'une solution locale, l'existence globale s'en déduisant facilement à partir des estimations a priori uniformes obtenues.

Soit $I^{\prime}=\left[0, T^{\prime}\right]$ un sous-intervalle de $I=[0, T]$ à déterminer. On adopte un pas constant $h=\frac{T^{\prime}}{n}$, donc les nouds $t_{i}^{n}=i h=i \frac{T^{\prime}}{n}$, en particulier $t_{0}^{n}=0, t_{n}^{n}=T^{\prime}$. Soit $v_{0}$ donné appartenant à $E$. Pour chaque $n$, on construit les suites approximantes finies $\left(v_{i}^{n}\right)$ et $\left(q_{i}^{n}\right)$ de $E$ :

$$
\begin{aligned}
& q_{0}^{n}=q_{0} \\
& v_{0}^{n}=-\left(e v_{0}+(1+e) \operatorname{prox}\left(V\left(q_{0}^{n}\right), v_{0}+\frac{h}{1+e} p\left(t_{0}^{n} \cdot q_{0}^{n}\right)\right)\right.
\end{aligned}
$$

et pour $i=0,1, \ldots, n-1$ :

$$
\begin{aligned}
& q_{i+1}^{n}=q_{i}^{n}+h v_{i}^{n}, \\
& v_{i+1}^{n}=-e v_{i}^{n}+(1+e) \operatorname{prox}\left(V\left(q_{i+1}^{n}\right), v_{i}+\frac{h}{1+e} p\left(t_{i+1}^{n}, q_{i+1}^{n}\right)\right) .
\end{aligned}
$$

On définit alors les fonctions en escalier approchantes :

$$
\begin{aligned}
& \left\{\begin{aligned}
v_{n}(t) & =v_{i}^{\prime \prime} & & \text { si } t \in\left[t_{i}^{n}, t_{i+1}^{n}[, \quad 0 \leq i \leq n-1 .\right. \\
& =v_{n}^{\prime \prime} & & \text { si } t=T^{\prime} .
\end{aligned}\right. \\
& q_{n}(t)=q_{0}+\int_{0}^{t} v_{n}(\tau) \mathrm{d} \tau
\end{aligned}
$$

et

Étape 1. - On montre la majoration

$$
\left|v_{i}^{n}\right| \leq R=\left|v_{0}\right|+2 T^{\prime} M
$$

où $M=\sup \left\{p(t, q(t)) ; t \in[0, T],\left|q(t)-q_{0}\right| \leq \delta\right\}$. L'application $q \longmapsto V(q)$ étant semi-continue inférieurement, il existe $\delta>0$ et une boule $\mathrm{B}(a, r)$ tels que $: V(q) \supset \mathrm{B}(a, r)$ pour $\left|q-q_{0}\right| \leq \delta$. On choisit alors $T^{\prime}$ par l'équation $T^{\prime}=\frac{\delta}{R}$, d'où : $\left|q_{i}^{n}-q_{0}\right| \leq \delta$ et $V\left(q_{i}^{n}\right) \supset \mathrm{B}\left(a, r^{\prime}\right), \forall n \in \mathbb{N}$, $\forall i \in\{0, \ldots, n-1\}$.

Étape 2. - Si $f \in \mathrm{C}^{1,1 / 2}(Q, \mathbf{R})$, on a la majoration des mesures $\mathrm{d} v_{n}$ :

$$
\sup _{n} \int_{\left[0, T^{\prime}\right]}\left|\mathrm{d} v_{n}\right|<+\infty .
$$


Par le théorème de Helly, la suite $\left(v_{n}\right)$ converge partout vers une fonction à variation bornée $v$. La suite $\left(q_{n}\right)$ converge alors uniformément vers la fonction $t \longmapsto q(t)=q_{0}+\int_{0}^{t} v(\tau) \mathrm{d} \tau$ et vérifie la relation $f(q(t)) \leq 0$.

Soit alors $u=\frac{v^{+}+e v^{-}}{1+e}$. Par construction, $u$ est lbve-m.

Étape 3. - Si $f \in \mathrm{C}^{1,1 / 2}(Q, R)$, on montre que $u$ vérifie presque partout l'équation (2). Ceci se fait en démontrant la relation intégrale :

$$
z \cdot(v(t)-v(s)) \geq \frac{1}{2}\left(|v(t)|^{2}-|v(s)|^{2}\right)+\int_{s}^{t}(z-v(\tau)) \cdot p(\tau, q(\tau)) \mathrm{d} \tau
$$

où $z \in V(y)$ pour tout $y$ appartenant à un voisinage de l'image $q([s, t]), s \leq t \leq T^{\prime}$, et en passant à la limite par le théorème de Jeffery sur la dérivation des mesures.

Étape 4. - $\mathrm{Si} f \in \mathrm{C}^{1, \beta}(Q, \mathbb{R}), \beta>1 / 2$, on montre que $u$ vérifie la relation de saut en tout point de discontinuité.

\section{Conclusion}

Les preuves des points sus-mentionnés sont très techniques et reposent sur des arguments analogues à ceux utilisés dans [2]. Le fait que $e$ soit non nul complique très sérieusement les démonstrations et nécessite plus de régularité pour $f$. Ceci peut s'interpréter comme une condition sur la courbure de la surface $f=0$.

Pour $e=0$ et $p=p(t, q(t))$, la condition $f \in \mathrm{C}^{\mathrm{l}}(Q, \mathbb{R})$ suffit, et nous retrouvons le résultat d'existence établi par M. Monteiro-Marques dans [2].

Si le système est soumis à des efforts dépendant aussi de la vitesse (amortissement visqueux par exemple) $p=p(t, q(t), \dot{q}(t))$, globalement continus, et lipschitziens par rapport à $q$ et $\dot{q}$, uniformément en $t$, le résultat d'existence demeure vrai. Seule l'étape 1 est à modifier substantiellement, les étapes 2 , 3 et 4 ne subissant que des changements mineurs.

Les preuves détaillées de cette Note sont à paraître prochainement dans [1].

\section{Références bibliographiques}

[1] Mabrouk M., On an unified variational model for the dynamics of unilateral constraints, Europ. J. Mech., (à paraître).

[2] Monteiro-Marques M.D.P., Chocs inélastiques standard : un résultat d'existence, Séminaire d'analyse convexe de Montpellier, exposé $n^{\circ} 4,1985$.

[3] Moreau J.J., Décomposition orthogonale d'un espace hilbertien selon deux cônes mutuellement polaires, C. R. Acad. Sci. Paris t. 255 (1962).

[4] Moreau J.J., Standard inelastic shocks and the dynamics of Unilateral constraints, In: Unilateral Problems in structural Analysis, Del Piero G., Maceri F. (Eds.), 1985.

[5] Moreau J.J., Unilateral contact and dry friction in finite freedom dynamics, Moreau J.J., Panagiotopoulos P.D. (Eds.), Nonsmooth mechanics and applications, Springer-Verlag, 1988, pp. 1-82. 